$(\mathrm{p}=0.008)$. Among the adult patients, approximately a third were given a same day appointment with their GP, and a half were offered an appointment within 48 hours. However, $11.5 \%$ were told they would have to wait more than a week, ranging between 9.6\% and 16.0\% ( $p=0.009)$. 86.3\% of children were given same day appointments. $62.5 \%$ were aware of an alternative service that could treat their urgent health need; almost half cited their GP, yet only $33 \%$ attempted to contact them. MIUs were also cited as an alternative option; over half of respondents across the four EDs attended due to an injury. Only a fifth of respondents sought advice from a health website, while half had a long term health condition.

Conclusion The study indicates that attendance by a proportion of patients could be avoided. The convenience and relatively short wait associated with attendance at $A \& E$ is recognised by the public. Inability to access GPs in a timely manner is an issue, as is the perceived role of GPs. The large proportion of people attending with injuries suggests underutilisation of, or a need for more, MIUs. Health websites should be promoted, especially to those with long term conditions.

\section{OP46 COMPARISON OF SEPSIS RECORDING IN PRIMARY CARE ELECTRONIC HEALTH RECORDS AND LINKED HOSPITAL EPISODES AND MORTALITY DATA: POPULATION-BASED COHORT STUDY IN ENGLAND}

E Rezel-Potts ${ }^{*}$, M Gulliford. School of Population Health and Environmental Sciences, King's College London, London, UK

\subsection{6/jech-2020-SSMabstracts.46}

Background Sepsis is a life-threatening condition resulting from systemic infection. Hospital admissions and recorded deaths for sepsis appear to be increasing nationally, heightening the need for epidemiological studies of sepsis based on accurate and complete data recording across linked records. We aimed to compare the recording of fatal and non-fatal sepsis across primary care electronic health records, hospital episodes and death registrations in England.

Methods A cohort study was conducted including patients registered with the Clinical Practice Research Datalink (CPRD). We analysed linked data for 378 general practices with 1,183,594 patient-years of follow-up and 21,426 first episodes of sepsis from 2002-2017. We searched linked HES admitted patient care records for sepsis events in the 30 days before and after first CPRD diagnosis. We searched the linked ONS death data for patients with sepsis recorded as any cause of death then identified if these patients had sepsis events recorded in the CPRD and HES in the 30 days preceding date of death. We calculated incidence rates and trends in age-standardised incidence in each of the linked records.

Results Among the 21,426 patients with a first episode of sepsis in the CPRD, 4,482 (21\%) had a sepsis event in HES in the 30 days before or after. There were 4,872 patients with sepsis listed as any cause of death in the ONS death data; 2,564 (53\%) had a sepsis event recorded in the CPRD in the 30 days before the ONS date of death compared to $1,187(24 \%)$ in HES. The incidence of new episodes of sepsis was 18.10 per 1000 patient-years $(10.75$ - 28.57) in the CPRD and $7.02(2.83$ - 14.45) in HES.
The mortality rate was 4.12 per 1000 patient-years (1.15 10.41) in the ONS death data. ONS records had peaks in sepsis mortality in 2006 and 2015 which were not reflected in the CPRD and HES records which had similar trends from low to high incidence and steep rates of increase from 2012 to 2017.

Conclusion There was a lack of agreement across data sources for both fatal and non-fatal sepsis events, indicating that relying on singular sources could lead to biased estimates of incidence. Linked electronic health records from primary care, hospital care, and death certificates should be used where possible to increase the accuracy and completeness of epidemiological findings.

\section{Thursday 10 September}

\section{Life Course: Early Life}

\section{OP47 ADVERSE CHILDHOOD EXPERIENCES AND ADULT INFLAMMATION IN THE 1958 BRITISH BIRTH COHORT: COMPARING SINGLE ADVERSITY, CUMULATIVE RISK AND LATENT CLASS APPROACHES}

${ }^{1}$ RE Lacey*, ${ }^{1} S M$ Pinto Pereira, ${ }^{2} \mathrm{~L} L i,{ }^{3,4,5} \mathrm{~A}$ Danese. ${ }^{1}$ Research Department of Epidemiology and Public Health, UCL, London, UK; ${ }^{2}$ Population, Policy and Practice Research and Teaching Department, UCL Great Ormond Street Institute of Child Health, London, UK; ${ }^{3}$ Social, Genetic and Developmental Psychiatry Centre, KCL, London, UK; ${ }^{4}$ Department of Child and Adolescent Psychiatry, KCL, London, UK; ${ }^{5}$ National and Specialist CAMHS Clinic for Trauma, Anxiety and Depression, South London and Maudesley NHS Foundation Trust, London, UK

\subsection{6/jech-2020-SSMabstracts.47}

Background Adverse childhood experiences (ACEs) have been related to poorer health across the life course. Previous studies typically relied on cumulative risk scores or individual adversities measured through retrospective selfreports. However these approaches have important limitations. Cumulative risk scores assume equal weighting of adversities and the single adversity approach ignores the high probability that adversities co-occur. In contrast, latent class analysis (LCA) offers an alternative approach to operationalise ACEs that respects the clustering of adversities and may identify specific patterns of ACEs important for health outcomes. Furthermore, prospective and retrospective reports of ACEs show poor agreement. Therefore, it is important to compare findings based on prospective and retrospective measures in the same individuals. The aim of this study was to compare LCA, single adversity and cumulative risk approaches to operationalising ACEs with inflammation in mid-life, comparing prospectively and retrospectively-reported ACEs data.

Methods Using data on 8,810 members of the 1958 British birth cohort we investigated 12 ACEs - physical, psychological and sexual abuse, physical and emotional neglect, parental mental health problems, witnessing abuse, parental conflict, parental divorce, parental offending, parental substance misuse and parental death. LCA was applied to explore the clustering of prospectively and retrospectively reported ACEs separately. Associations between latent classes, cumulative risk scores and individual adversities with three inflammatory markers (CReactive Protein, fibrinogen and von Willebrand Factor) were tested using linear regression. 
Results There was co-occurrence between adversities, and particularly for retrospectively reported adversities. Three latent classes were identified in the prospective data - 'Low ACEs', 'Household dysfunction' (2.8\%) and 'Parental loss' $(1.5 \%)$ which were related to increased inflammation in mid-life, as were high cumulative risk scores and individual measures of offending, death, divorce, physical neglect and family conflict. Four latent classes were identified in the retrospective data - 'Low ACEs', 'Parental mental health and substance misuse', 'Maltreatment and conflict' and 'Polyadversity.' The latter two (5.2\%) were related to raised inflammation in mid-life, as was a retrospective ACE score of $4+(8.3 \%)$ and individual measures of family conflict, psychological and physical abuse, emotional neglect and witnessing abuse.

Discussion Specific ACEs or ACE combinations might be important for chronic inflammation. LCA is an alternative approach to operationalising ACEs data but further research is needed. Identifying the specific ACEs or combinations of ACEs which are most strongly related to inflammation is important for investigating the mechanisms involved and the planning of effective interventions.

\section{OP48 IS STATE SCHOOL EDUCATION REALLY FREE? LISTENING TO CHILDREN'S VIEWS ON THE COSTS OF THE SCHOOL DAY}

${ }^{1} \mathrm{~L}$ Naven*, ${ }^{2} \mathrm{E}$ Sosu, ${ }^{3} \mathrm{~S}$ Spencer, ${ }^{1} \mathrm{~J}$ Egan. ${ }^{1}$ Glasgow Centre for Population Health, Glasgow, UK; ${ }^{2}$ School of Education, University of Strathclyde, Glasgow, UK; ${ }^{3}$ Child Poverty Action Group, Glasgow, UK

\subsection{6/jech-2020-SSMabstracts.48}

Background In the context of child poverty and educational attainment in Scotland, the Cost of the School Day (CoSD) project privileged children's voices to examine the potential influence of school policies and practices on the ability of children from low-income families to participate fully in the school day.

Methods A purposive sample of Glasgow schools (4 primaries and 2 secondaries) was chosen, to ensure representation across the socio-economic spectrum using the Scottish Index of Multiple Deprivation. Pupils over 16 years could opt in to focus group sessions, and consent was sought from parents/carers of pupils under 16. Sessions used a vignette of a fictional character from a low-income household, allowing a safe degree of personal distance for pupils to explore sensitive topics. Groups, comprising pupils of mixed gender and free school meal entitlement, took part in two sessions. Initial sessions identified costs involved in attending school, and follow-up sessions considered potential actions needed by schools. 71 focus group sessions with 282 pupils were carried out. Deductive thematic analysis was carried out using QSR NVivo software.

Results Pupils identified substantial barriers to participation in the school day and suggested potential solutions. Key themes centered around transport costs, access to afterschool activities and fun events, curriculum costs for subjects and textbooks, home schoolwork resources and school uniform costs.

Pupils highlighted that transport costs present barriers to holiday revision classes and after-school clubs and activities, and suggested providing clubs and supported study at different times of the school day to ensure transport is not an issue.

Curriculum costs for subjects, textbooks and specimen papers were raised, as well as internet and computing requirements for homework. Groups felt that schools should offer more than one way of completing homework.

School uniform emerged as a major cost leading to stigma and embarrassment, absence, or exclusions for non-compliance. Pupils felt more support is needed to ensure affordability, schools should signpost to the cheapest uniform suppliers, and systems are needed to avoid pupils feeling embarrassed.

Conclusion The findings support the argument that the way the curriculum is structured and implemented is more advantageous to pupils from higher-income households. The CoSD tapped into the Scottish Government policy cycle and resulted in changes to increase the school clothing grant across Scotland, and, at Glasgow level, to automate clothing grant payments to ensure uptake. The CoSD lessons are transferrable across education systems and are being further rolled out in 128 schools across the UK.

\section{OP49 PERCEPTIONS OF ADOLESCENT HEALTH RISK BEHAVIOUR AND SOCIOECONOMIC POSITION: A GROUNDED THEORY STUDY OF YOUNG ADULTS IN BRISTOL}

L Tinner*, D Caldwell, M Hickman, R Campbell. Population Health Sciences, Bristol Medical School, University of Bristol, Bristol, UK

\subsection{6/jech-2020-SSMabstracts.49}

Background Health risk behaviours such as tobacco smoking, alcohol consumption, drug misuse, unhealthy diet and unprotected sexual intercourse are global health issues, often initiated in adolescence. There is contrasting evidence on the association between health risk behaviours and socioeconomic position in adolescence and young adulthood, with little qualitative evidence to illuminate the relationship. The aim of the study was to examine to what extent young people perceive health risk behaviours to shape socioeconomic inequalities while transitioning into adulthood.

Methods We undertook a nested qualitative study within The Avon Longitudinal Study of Parents and Children (ALSPAC) birth cohort using semi-structured interviews of 25 young adults aged 26-28 years. A grounded theory approach was adopted. Theoretical sampling was conducted based on participants' early life socioeconomic background and engagement in health risk behaviours when they were adolescents. Data collection and analysis were undertaken iteratively to aid constant comparison.

Results Categories of peer influence, family influence, 'drive to succeed' and personal responsibility were identified. These data underline the importance of family and peers in relation to both health risk behaviours and socioeconomic life chances as young people transition into adulthood. Engaging in some level of health risk behaviour allowed young people to gain experiences and bond with peers but avoiding certain behaviours helped construct their identity and self-security. Some participants adopted a moralistic or neoliberal perspective in labelling peers who engaged in certain risk behaviours, such as drug use, as irresponsible or immature. Adolescent alcohol use was seen as normative 